\title{
Nonsense-Mediated Decay
}

National Cancer Institute

\section{Source}

National Cancer Institute. Nonsense-Mediated Decay. NCI Thesaurus. Code C29648.

Nonsense-Mediated Decay is the widely conserved process in eukaryotes whereby certain alternatively spliced RNAs, some selenoprotein mRNAs, and nonsense or frameshift mutations that result in premature translation termination signal rapid mRNA degradation; the mechanism that eliminates mRNAs with premature termination codons, reducing the accumulation of deleterious proteins and their effects. NMD is initiated during translation when hUpf proteins phosphorylated by SMG proteins cooperate with an exon-junction complex to identify mRNAs with premature stop codons and mark the transcript for rapid decay. Degrading mRNAs from both ends, NMD involves decapping, deadenylating, and exonucleolytic activities. 